\title{
Agreement of Sedentary Behavior Metrics Derived From Hip- and Thigh-Worn Accelerometers Among Older Adults: With Implications for Studying Physical and Cognitive Health
}

\author{
John Bellettiere and \\ Fatima Tuz-Zahra \\ University of California San Diego \\ Sandy Liles \\ University of California San Diego
}

\author{
Jordan A. Carlson \\ Children's Mercy Hospital and \\ University of Missouri-Kansas City \\ Mikael Anne Greenwood- \\ Hickman and Rod L. Walker \\ Kaiser Permanente Washington \\ Health Research Institute
}

\author{
Nicola D. Ridgers \\ Deakin University
}

\author{
Andrea Z. LaCroix and \\ Marta M. Jankowska \\ University of California San Diego
}

\author{
Loki Natarajan \\ University of California San Diego
}

\begin{abstract}
Little is known about how sedentary behavior (SB) metrics derived from hip- and thigh-worn accelerometers agree for older adults. Thigh-worn activPAL (AP) micro monitors were concurrently worn with hip-worn ActiGraph (AG) GT3X+ accelerometers (with SB measured using the 100 counts per minute [cpm] cut point; $\mathrm{AG}_{100 \mathrm{cpm}}$ ) by 953 older adults (age $77 \pm 6.6,54 \%$ women) for 4-7 days. Device agreement for sedentary time and five SB pattern metrics was assessed using mean error and correlations. Logistic regression tested associations with four health outcomes using standardized (i.e., $z$ scores) and unstandardized SB metrics. Mean errors $\left(\mathrm{AP}-\mathrm{AG}_{100 \mathrm{cpm}}\right)$ and $95 \%$ limits of agreement were: sedentary time $-54.7[-223.4,113.9] \mathrm{min} / \mathrm{day}$; time in 30+ min bouts $77.6[-74.8,230.1] \mathrm{min} /$ day; mean bout duration $5.9[0.5,11.4] \mathrm{min}$; usual bout duration $15.2[0.4,30] \mathrm{min}$; breaks in sedentary time $-35.4[-63.1,-7.6]$ breaks/day; and alpha $-.5[-.6,-.4]$. Respective Pearson correlations were: .66, .78, .73, .79, .51, and .40. Concordance correlations were: $.57, .67, .40, .50, .14$, and .02 . The statistical significance and direction of associations were identical for $\mathrm{AG}_{100 \mathrm{cpm}}$ and $\mathrm{AP}$ metrics in 46 of 48 tests, though significant differences in the magnitude of odds ratios were observed among 13 of 24 tests for unstandardized and five of 24 for standardized SB metrics. Caution is needed when interpreting SB metrics and associations with health from $\mathrm{AG}_{100 \mathrm{cpm}}$ due to the tendency for it to overestimate breaks in sedentary time relative to AP. However, high correlations between $\mathrm{AP}$ and $\mathrm{AG}_{100 \mathrm{cpm}}$ measures and similar standardized associations with health outcomes suggest that studies using $\mathrm{AG}_{100 \mathrm{cpm}}$ are useful, though not ideal, for studying SB in older adults.
\end{abstract}

Keywords: frailty and physical function, physical activity, sedentary accumulation patterns, sedentary behavior patterns, sitting time

Sedentary behavior is increasing in modern society, and among older adults, it is the most prevalent behavior among sleep, sedentary behavior, and physical activity. (Diaz et al., 2016; Du et al., 2019; Jefferis et al., 2015; Matthews et al., 2012; Yang et al., 2019) By consensus, sedentary behavior has been defined as all

Rosenberg and Natarajan are co-senior authors. Bellettiere, Tuz-Zahra, Liles, LaCroix, and Natarajan are with the Herbert Wertheim School of Public Health and Human Longevity Science, University of California San Diego, La Jolla, CA, USA. Carlson is with the Center for Children's Healthy Lifestyles and Nutrition, Children's Mercy Hospital, Kansas City, MO, USA; and the Department of Pediatrics, Children's Mercy Hospital and University of Missouri, Kansas City, Kansas City, MO, USA. Ridgers is with the Institute for Physical Activity and Nutrition, School of Exercise and Nutrition Sciences, Deakin University, Geelong, Victoria, Australia. Greenwood-Hickman, Walker, and Rosenberg are with the Kaiser Permanente Washington Health Research Institute, Seattle, WA, USA. Jankowska is with the Qualcomm Institute/Calit2, University of California San Diego, La Jolla, CA, USA. Bellettiere (jbellettiere@ ucsd.edu) is corresponding author. waking behaviors while in a seated or lying posture that result in an energy expenditure $\leq 1.5$ metabolic equivalents (Tremblay et al., 2017). When measured using ActiGraph (AG) accelerometers worn around the participant's hip, sedentary time is estimated based on lack of movement and does not factor in posture. To obtain device-based measures of sedentary behavior derived from both posture and movement, the most common approach is to attach an activPAL (AP) monitor-an inclinometer/accelerometer specifically designed as a thigh-worn device capable of assessing posture and thus capturing sitting/lying - to the participant's thigh (Edwardson et al., 2016). This approach is often viewed as the device-based standard for the measurement of sedentary behavior.

Accelerometers produce time-stamped data, making it possible to identify when during the day activity occurs (Glazer et al., 2013; Lord et al., 2011), as well as the patterns in which behaviors are accumulated (Chastin \& Granat, 2010). These patterns include the timing, frequency, and duration of sedentary bouts and breaks throughout the day (Tremblay et al., 2017). While there has been an 
increase in the number of studies examining sedentary patterns and their associations with health (e.g., Brocklebank, Falconer, Page, Perry, \& Cooper, 2015; Jefferis et al., 2019), the Physical Activity Guidelines for Americans, 2nd edition (U.S. Department of Health and Human Services, 2018), and the update (Katzmarzyk et al., 2019) both highlighted that further research using prospective cohorts to study sedentary patterns in relation to mortality and other health outcomes is needed.

One challenge to updating this literature is that most prospective cohort studies use hip-worn AG accelerometers rather than the thigh-worn AP monitor (Lee \& Shiroma, 2014), and the measurement of sedentary bouts and breaks using hip-worn accelerometers and standard data processing techniques (Migueles et al., 2017) is not accurate (Barreira, Zderic, Schuna, Hamilton, \& Tudor-Locke, 2015). For example, when compared with direct observation of sit-to-stand transitions during usual free-living conditions, one study of 13 adults reported $0.3 \%$ bias for thigh-worn AP-measured transitions and $98.6 \%$ bias for hip-worn AG-measured transitions (Lyden, Kozey Keadle, Staudenmayer, \& Freedson, 2012). Despite the inaccuracy, the authors reported correlation coefficients compared with direct observation of .97 for the AP-measured transitions and .92 for the AG-measured transitions (Lyden et al., 2012). There has also been evidence of convergent validity in several labs and cohorts around the world that showed AG-derived measures of sedentary patterns were associated with various health outcomes in the expected direction (Bellettiere et al., 2019; Brocklebank et al., 2015; Diaz, Goldsmith, et al., 2017; Diaz, Howard, et al., 2017). Given the historical and continuing use of AG accelerometers in prospective cohorts, understanding the agreement between AG- and posture-based measures for assessing sedentary behavior, including accumulation patterns, is critical for advancing the field.

The aim of this study was to assess agreement between the most commonly used hip-worn AG measures of volumes and patterns of sedentary time and thigh-worn AP measures in a well-characterized cohort of older adults and determine how any measurement error may bias associations of sedentary behavior with physical or cognitive health.

\section{Methods}

In 1994, adults over 65 without dementia were randomly sampled from the King County membership of Group Health Cooperative of Puget Sound (now Kaiser Permanente Washington) to join the Adult Changes in Thought (ACT) study, a longitudinal cohort study of aging and incident dementia. An expansion cohort was enrolled starting in 2000. In 2005, a cohort refreshment protocol to maintain a constant 2000 participants was initiated by enrolling new members to replace those who died, were diagnosed with dementia, or dropped out of the study. Beginning in April 2016, enrolled participants were given the option to wear an AG GT3X+ accelerometer (ActiGraph LLC, Pensacola, FL) and/or an AP micro (PAL Technologies, Glasgow, Scotland, United Kingdom). Of the 1688 eligible participants who were able to and asked to wear devices, 1,151 wore the AG (1,088 returned devices with four or more adherent days, which for both devices were defined as days having 10 or more hours of awake wear), and 1,135 wore the AP (1,039 returned devices with at least four adherent days). The 953 men and women who wore both devices concurrently for at least four adherent days comprised our study population. Details on the ACT cohort and accelerometer deployment are published elsewhere (Rosenberg et al., 2020). Ethics approval was obtained from the Kaiser Permanente Washington Institutional Review Board, and all participants provided written informed consent.

\section{Accelerometers}

Participants were asked to wear devices for the same 7 days and to complete sleep logs each night of wear to document their in-bed and out-of-bed times. Sleep-log data were double entered to protect against transcription errors. Missing data were imputed using person-specific means if available and ACT sample means otherwise. Recorded in-bed and out-of-bed times were used to identify awake time for processing data from both devices.

The AG GT3X+ was worn $24 \mathrm{hr} /$ day on an elastic waistband at the right hip region with data collected at $30 \mathrm{~Hz}$. Using ActiLife (version 6.13.3; ActiGraph LLC, Pensacola, FL), 15-s epoch data were generated using the normal filter then aggregated to 1-min epochs. Device nonwear was determined using the Choi algorithm (Choi, Liu, Matthews, \& Buchowski, 2011; Choi, Ward, Schnelle, \& Buchowski, 2012) applied to vector magnitude counts per minute (CPM) using a 90-min window, 30-min streamframe, and 2-min tolerance. To classify awake-wear-time epochs as sedentary, the most common cut point (100 CPM) was applied to the vertical axis (Migueles et al., 2017). Sedentary bouts were then derived as consecutive waking sedentary time epochs with no minimum and no tolerance.

The AP3 micro was placed in a waterproof casing and secured to the center of the right thigh using Tegaderm adhesive tape (3M, St. Paul, MN). Using the default setting in palBatch (version 7.2.32; PAL Technologies, Glasgow, Scotland, UK), data were converted to event-level files which were then visually inspected for anomalies using heat maps that had sleep-log and AG data superimposed. Sitting events that occurred while participants were awake (a.k.a., sitting bouts) were used to compute sedentary behavior metrics.

\section{Sedentary Behavior Metrics}

Sedentary behavior metrics were computed using only adherent days on which both devices were worn. Total sedentary time was averaged across all adherent days for each device. Five of the most commonly used sedentary accumulation pattern metrics were evaluated. Time spent in $30+$ min bouts was computed as the average sedentary time per day that was accumulated in bouts that were $30 \mathrm{~min}$ in duration or greater. Mean bout duration was computed as the sum of all sedentary bout durations divided by the number of bouts. The usual bout duration (UBD) and alpha were computed using the methods described by Chastin and colleagues (Chastin \& Granat, 2010; Chastin et al., 2015). UBD is the midpoint of the cumulative distribution of sedentary bout durations, computed using nonlinear regression. UBD indicates the bout duration above which half of all sedentary time is accumulatedhigher values indicate a more prolonged accumulation pattern. Alpha characterizes the shape of the sedentary bout duration distribution for each person with lower alphas reflecting frequent long bouts with few short bouts and higher alphas reflecting frequent short bouts with few long bouts (see Supplementary Figure 1 in our earlier publication for more details [Bellettiere et al., 2017]). The number of breaks was computed as the average number of sedentary bouts per day over all adherent days. In this regard, sedentary breaks represented the number of times per day there was a transition from a sedentary bout to movement above the $100 \mathrm{cpm}$ cut point, while sitting breaks represented the number of 
times per day there was a transition from a sitting/lying posture to standing or stepping.

\section{Outcomes}

At the ACT study visit when accelerometers were distributed, participants' height and weight were measured by trained staff using a tape measure and the clinic scale. Body mass index (BMI) was computed as the weight (in kilogram) divided by height (in meter square). Participants also completed questionnaires. From the RAND 36 questionnaire (Ware, 2000; Ware \& Sherbourn, 1992), self-rated health was assessed with a single item asking "In general, would you say your health is: excellent, very good, good, fair, poor?"; difficulty walking half a mile was assessed with a single item asking "Does your health now limit you in walking half a mile and if so, how much?" Responses were yes, limited a lot; yes, limited a little; and no, not limited at all. Global cognitive performance was measured using the Cognitive Abilities Screening Instrument, which consists of a short series of tests that assess nine domains of cognitive impairment (Chiu, Yip, Woo, \& Lin, 2019; De Oliveira et al., 2016; Teng et al., 1994). Resulting scores range from 0 to 100 (best functioning). Cognitive impairment was defined as having a score $\leq 86$ on the Cognitive Abilities Screening Instrument and/or referral for additional diagnostic workup. For analyses, BMI, self-rated health, difficulty walking a half mile, and cognitive function-selected for analysis because of known or expected associations with sedentary behavior-were dichotomized as $\geq 30$ versus $<30$; good, poor, and very poor versus very good and excellent; yes versus no; and cognitive impairment, yes versus no, respectively.

\section{Statistical Methods}

Summary statistics described distributions of participant characteristics. Histograms were plotted to show distributions of the six sedentary behavior metrics separately for AP and $\mathrm{AG}_{100 \mathrm{cpm}}$ mea-

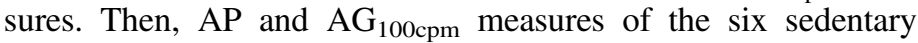
behavior metrics were compared at the person level by computing mean error ( $\left.\mathrm{AP}-\mathrm{AG}_{100 \mathrm{cpm}}\right)$ and mean absolute error. Agreement between the two devices was visually assessed using the BlandAltman approach, with $95 \%$ limits of agreement computed using regression analysis to test for and account for relationships between bias and magnitude (Bland \& Altman, 2007). Pearson and Spearman correlation coefficients were used to describe the betweendevice linear relationship of the sedentary behavior metrics. Concordance correlation coefficients were used to assess the degree to which the between-device linear relationship aligns with the $45^{\circ}$ line. To explore the epidemiologic implications of any potential measurement error, associations of the six sedentary behavior metrics with four indicators of health (self-rated health, BMI, difficulty walking half a mile, and cognitive impairment) were estimated using separate multivariable logistic regression models. Values for all sedentary behavior metrics were first converted to $z$ scores to standardize the resulting beta coefficients. This enabled a distribution-based "apples-to-apples" between-device comparison of the size of the odds ratios (ORs) as the underlying unit of change in each $z$-score-converted sedentary behavior metric is identical (i.e., a $1 S D$ increment). Models were adjusted for age, gender, race/ethnicity (Hispanic or non-White vs. non-Hispanic White), and education (less than high school, completed high school, some college, or completed college). The resulting standardized ORs from models using $\mathrm{AG}_{100 \mathrm{cpm}}$ sedentary behavior metrics were compared with the corresponding standardized ORs from models using AP metrics, according to the methods described by Horton and Fitzmaurice (2004). Results from analyses of sedentary behavior and health outcomes were also reported using unstandardized ORs, which were computed using sedentary behavior metrics that were not converted to $z$ scores and instead remained in their original units of measure.

Following initial peer review, we reran agreement analyses after classifying sedentary behavior using alternate cut points of $200 \mathrm{cpm}$ applied to the vector magnitude and $25 \mathrm{cpm}$ applied to the vertical axis (Aguilar-Farías, Brown, \& Peeters, 2014), cut points that were similar to those reported by Koster et al. (2016).

Analyses were conducted using R (R Foundation for Statistical Computing, Vienna, Austria) with two-tailed statistical tests and statistical significance set to $p<.05$.

\section{Results}

The 953 ACT participants included in this study were, on average, aged $77 \pm 7$ years, $54.3 \%$ female, $87.0 \%$ non-Hispanic White, and $72.7 \%$ completed college (Table 1 ).

Distributions for each sedentary behavior metric separately for $\mathrm{AP}$ and $\mathrm{AG}_{100 \mathrm{cpm}}$ measures are presented in Supplementary

\section{Table 1 Participant Characteristics for Men and Women of the ACT Study Who Concurrently Wore AG and AP Accelerometers; $N=953$}

\begin{tabular}{lc}
\hline Quantitative variables, mean $(S D)$ & $77(6.6)$ \\
Age (years) & \\
Categorical variables, $n(\%)$ & \\
Gender & $421(44.2 \%)$ \\
$\quad$ Men & $532(55.8 \%)$ \\
Women & \\
Race/ethnicity & $97(10.2 \%)$ \\
Hispanic or non-White & $853(89.5 \%)$ \\
non-Hispanic White & $15(1.6 \%)$ \\
Education & $74(7.8 \%)$ \\
$\quad$ Less than high school & $152(16.0 \%)$ \\
Completed high school & $712(74.7 \%)$ \\
Some college & \\
Completed college & $722(75.8 \%)$ \\
BMI & $212(22.2 \%)$ \\
BMI below 30 & \\
BMI 30 or above & \\
Self-rated health & $599(62.9 \%)$ \\
Very good and excellent & $354(37.1 \%)$ \\
Good, poor, or very poor & \\
Difficulty in walking half a mile & \\
None & $226(76.2 \%)$ \\
Some & $227(23.8 \%)$ \\
Impgnitive impairment & \\
Not impaired & \\
\hline
\end{tabular}

Note. $\mathrm{AP}=$ activPAL; $\mathrm{AG}=$ ActiGraph ACT $=$ Adult Changes in Thought BMI $=$ body mass index. Percentages may not sum to $100 \%$ due to rounding. 
Table 2 Summary and Agreement Results for Sedentary Time and Patterns of Sedentary Time Measured Using AG GT3X+ and AP Micro Among ACT Participants; $N=953$

\begin{tabular}{|c|c|c|c|c|c|c|}
\hline Statistic & $\begin{array}{l}\text { Total sedentary } \\
\text { time (min/day) }\end{array}$ & $\begin{array}{l}\text { Time spent in } 30+ \\
\text { min bouts } \\
\text { (min/day) }\end{array}$ & $\begin{array}{c}\text { Mean bout } \\
\text { duration (min) }\end{array}$ & UBD (min) & $\begin{array}{c}\text { Number of } \\
\text { breaks in } \\
\text { sedentary time }\end{array}$ & Alpha \\
\hline $\mathrm{AP}$, mean $(S D)$ & $598.4(116.1)$ & $351.2(135.5)$ & $14.8(6.6)$ & $37.9(17.8)$ & $44.5(12.8)$ & $1.30(.04)$ \\
\hline $\mathrm{AG}$, mean $(S D)$ & $653.2(95.2)$ & $273.6(128.0)$ & $8.9(3.9)$ & $22.7(12.5)$ & $79.9(18.1)$ & $1.79(.13)$ \\
\hline \multicolumn{7}{|l|}{ Person-level agreement } \\
\hline Mean error $(A P-A G)$ & -54.7 & 77.6 & 5.9 & 15.2 & -35.4 & -.49 \\
\hline $95 \%$ LoA & {$[-223.4,113.9]$} & {$[-74.8,230.1]$} & {$[0.5,11.4]$} & {$[0.4,30.0]$} & {$[-63.1,-7.6]$} & {$[-.6,-.4]$} \\
\hline Mean absolute error & 80.1 & 92.9 & 6.1 & 15.7 & 35.3 & .49 \\
\hline Pearson correlation & $.66[.62, .70]$ & $.78[.76, .81]$ & $.73[.7, .76]$ & $.79[.77, .82]$ & $.51[.46, .56]$ & $.40[.34, .45]$ \\
\hline Concordance correlation & $.57[.53, .61]$ & $.67[.64, .70]$ & $.40[.37, .43]$ & $.50[.47, .53]$ & $.14[.12, .15]$ & $.02[.01, .02]$ \\
\hline
\end{tabular}

Note . $\mathrm{ACT}=$ Adult Changes in Thought; $\mathrm{AP}=$ activPAL; $\mathrm{AG}=$ ActiGraph; $\mathrm{LoA}=$ limits of agreement; $\mathrm{UBD}=$ usual bout duration.

Figure 1 (available online). Mean total sedentary time as measured by $\mathrm{AG}_{100 \mathrm{cpm}}(653 \mathrm{~min} /$ day) was $9.1 \%$ higher compared with the AP (598 min/day), resulting in a mean difference of $54.7 \mathrm{~min} /$ day (limit of agreement $[\mathrm{LOA}]=-223.4,113.9$; Table 2). There were nearly twice as many breaks in sedentary time detected by the $\mathrm{AG}_{100 \mathrm{cpm}}$ (mean $=79.9$ breaks/day) than the $\mathrm{AP} \quad($ mean $=$ 44.5 breaks/day; mean difference $=35.4$ breaks $/$ day $;$ LOA $=[-63.1$, -7.6]). The higher number of breaks recorded using $A_{100 \mathrm{cpm}}$ resulted in 77.6 fewer minutes per day $(\mathrm{LOA}=[-74.8,230.1])$ by $\mathrm{AG}_{100 \mathrm{cpm}}$ than $\mathrm{AP}$ in time spent in $30+$ min sedentary bouts. The mean difference in mean bout durations was 5.9 min $(\mathrm{LOA}=$ $[0.5,11.4] \mathrm{min})$, resulting in a $39.9 \%$ shorter mean when measured by $\mathrm{AG}_{100 \mathrm{cpm}}(8.9 \mathrm{~min})$ than by $\mathrm{AP}(14.8 \mathrm{~min})$. The mean difference in UBDs was $15.2 \mathrm{~min}\left(\mathrm{UBD}_{\mathrm{AG}}=22.7 \mathrm{~min}, \mathrm{UBD}_{\mathrm{AP}}=37.9 \mathrm{~min}\right)$, resulting in a $40.1 \%$ shorter mean. The higher frequency of and generally shorter duration of bouts detected by $\mathrm{AG}_{100 \mathrm{cpm}}$ resulted in a more rapid decay of the bout duration distribution and higher alpha values $\left(\right.$ alpha $_{\mathrm{AG}}=1.3$, alpha $\left.\mathrm{AP}=1.8\right)$. Bland-Altman plots illustrating mean differences for each metric are shown in Supplementary Figure 2 (available online).

Pearson correlations between $\mathrm{AG}_{100 \mathrm{cpm}}$ and $\mathrm{AP}$ for all sedentary behavior variables ranged between .40 and .79. Sedentary breaks and alpha had lower correlations $(r=.40$ and $r=.51$, respectively) than total sedentary time $(r=.66)$, time in long bouts $(r=.78)$, mean bout duration $(r=.73)$, and UBD $(r=.79)$. Spearman correlations produced similar results (data not shown). Concordance correlation coefficients followed a similar pattern ranging from .02 to .67. Alpha and sedentary breaks had lower concordance correlations (.02 and .14, respectively) compared with total sedentary time (.57), time in long bouts (.67), mean bout duration (.40), and USD (.50).

Measures of total sedentary time using the Aguilar-Farías et al. (2014) cut points were, on average, lower than AP-measured sedentary time, with $95 \%$ limits of agreement contained the value of zero, and a slightly lower mean error (see Supplementary Table 1 [available online]). Total sedentary time correlation coefficients for $\mathrm{AG}_{25 \mathrm{cpm}}(r=.70$ and concordance correlation $=.64)$ and $\mathrm{AG}_{200 \mathrm{cpm}}$ $(r=.80$ and concordance correlation $=.77)$ were higher than those for $\mathrm{AG}_{100 \mathrm{cpm}}(r=.66$ and concordance correlation =.57). Agreement for sedentary accumulation pattern metrics were not appreciably different when processing data using the Aguilar-Farías et al. (2014) cut points versus the $100 \mathrm{cpm}$ cut point, though more similarities were observed for the $200 \mathrm{cpm}$ vector magnitude cut point than for the $25 \mathrm{cpm}$ vertical axis cut point.
Figure 1 shows standardized ORs and 95\% confidence intervals (CIs) for associations between sedentary behavior and health. Lower self-rated health (i.e., good, poor, or very poor) was significantly related to all sedentary behavior metrics regardless of the device used, and there were no statistically significant differences between the computed ORs for $\mathrm{AG}_{100 \mathrm{cpm}}$ and $\mathrm{AP}$ measures. While not significantly different for all metrics, point estimates for ORs were generally stronger when computed using AP measures than when using $A_{100 \mathrm{cpm}}$ measures of total sedentary time, $30+\mathrm{min}$ bouts, mean bout duration, and UBD; OR point estimates were slightly larger when computed using $\mathrm{AG}_{100 \mathrm{cpm}}$ measures of alpha and sedentary breaks. The same general pattern was observed for associations with BMI, in that differences in ORs between AG and AP were significant for total sedentary time $(p<.001), 30+$ min bouts ( $p \leq .001)$, mean bout duration $(p=.01)$, and usual bout duration $(p=.03)$. For example, a $1 S D$ increment in total sedentary time measured by AP was associated with 1.94 times higher odds of BMI $\geq 30$ compared with BMI $<30(95 \%$ CI $[1.63,2.32])$, whereas a $1 S D$ increment in $\mathrm{AG}_{100 \mathrm{cpm}}$ total sedentary time resulted in $\mathrm{OR}(95 \% \mathrm{CI})$ of $1.56[1.32,1.85]$. Difficulty walking half a mile was associated with all six sedentary behavior metrics. The only significant difference between $\mathrm{AG}_{100 \mathrm{cpm}}$ and AP OR point estimates was observed for alpha $(p<.001)$, with a greater magnitude $\mathrm{OR}$ for $\mathrm{AG}(\mathrm{OR}=0.55 ; 95 \% \mathrm{CI}$ $[0.46,0.67])$ than for AP $(\mathrm{OR}=0.77 ; 95 \%$ CI $[0.65,0.91])$. For cognitive impairment, there were no statistically significant associations with any of the sedentary behavior metrics and no significant differences in ORs between $\mathrm{AG}_{100 \mathrm{cpm}}$ and AP.

When metrics were not standardized, significant differences in ORs between $\mathrm{AG}_{100 \mathrm{cpm}}$ and $\mathrm{AP}$ measures were more common (Figure 2). For example, associations of all six sedentary behavior metrics with BMI differed significantly between devices. For all four health outcomes, the largest differences were observed for mean bout duration, with higher ORs for $\mathrm{AG}_{100 \mathrm{cpm}}$ measures than AP measures. Alpha was consistently differentially associated with three outcomes, with stronger ORs for AP than $A_{100 \mathrm{cpm}}$.

\section{Discussion}

In this investigation of sedentary behavior metrics computed using data from concurrently worn AG and AP devices among older adults, agreement was generally highest for the two metrics that relied most heavily on the volume of sedentary behavior: total sedentary time and time in $30+$ min bouts. Our data showed that the 
(a) Associations with self-rated health (good, poor, and very poor vs. very good and excellent)

\begin{tabular}{lcccc} 
& $\mathrm{OR}_{\mathrm{AP}}$ & $\mathrm{OR}_{\mathrm{AG}}$ & $p$ value \\
\hline Total sedentary time & $1.30[1.13,1.50]$ & $1.21[1.05,1.40]$ & .70 & .40 \\
Time spent in bouts $\geq 30 \mathrm{~min}$ & $1.36[1.18,1.57]$ & $1.28[1.10,1.48]$ & .46 & .46 \\
Mean bout duration & $1.36[1.18,1.59]$ & $1.27[1.10,1.50]$ & .32 \\
Usual bout duration & $1.33[1.15,1.56]$ & $1.25[1.08,1.47]$ & .16 & \\
Average breaks in sed bouts & $0.87[0.75,1.00]$ & $0.84[0.72,0.96]$ & 0.75 & 1 \\
Alpha & $.85[.74, .98]$ & $.82[.71, .95]$ & & 1.25
\end{tabular}

(b) Associations with body mass index ( $\geq 30$ vs. $<30$ )

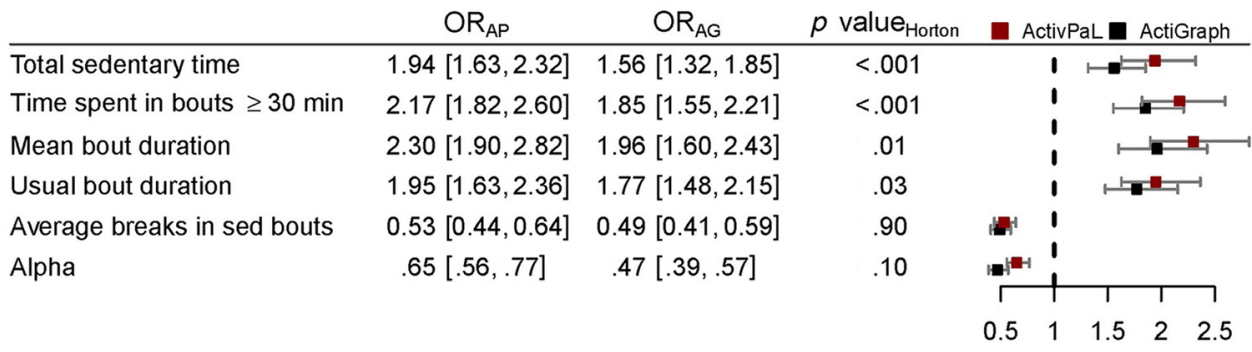

(c) Associations with difficulty walking half mile (any vs. none)

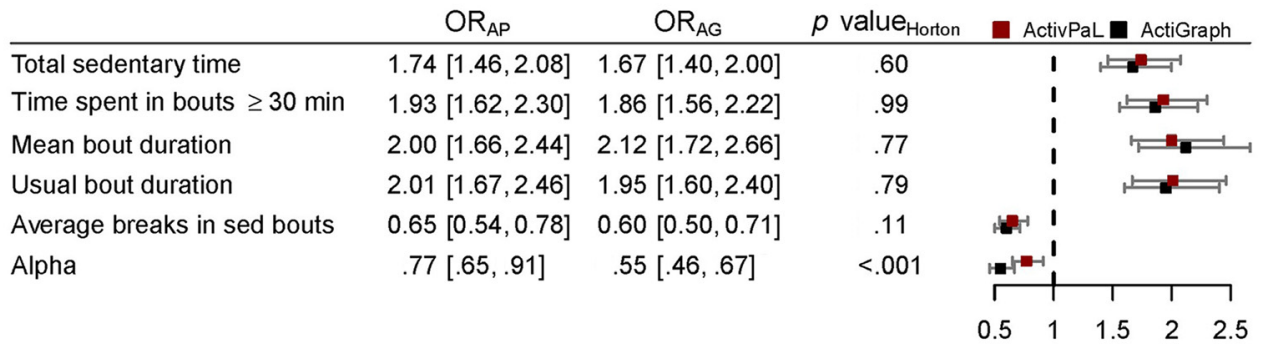

(d) Associations with cognitive impairment (impaired vs. not impaired)

\begin{tabular}{lcccc} 
& $\mathrm{OR}_{\mathrm{AP}}$ & $\mathrm{OR}_{\mathrm{AG}}$ & $p$ value $\mathrm{Horton}_{\text {ActivPaL ActiGraph }}$ \\
\hline Total sedentary time & $0.94[0.58,1.52]$ & $0.91[0.55,1.48]$ & .77 \\
Time spent in bouts $\geq 30 \mathrm{~min}$ & $1.13[0.71,1.76]$ & $1.12[0.71,1.72]$ & .91 \\
Mean bout duration & $1.26[0.90,1.66]$ & $1.30[0.98,1.65]$ & .74 \\
Usual bout duration & $1.17[0.84,1.51]$ & $1.30[0.94,1.68]$ \\
Average breaks in sed bouts & $0.86[0.55,1.33]$ & $0.88[0.58,1.35]$ & .93 \\
Alpha & $1.13[.70,1.88]$ & $.93[.57,1.48]$ & .08 & 0.5
\end{tabular}

Figure 1 - Forest plots for standardized (based on a $1 S D$ unit change in each metric) associations of $A_{100 c p m}$ and AP sedentary behavior metrics with self-rated health (a), BMI (b), difficulty walking half mile (c), and cognitive impairment (d). All models are adjusted for age, sex, race/ethnicity, and education. The $p$ value Horton $_{1}$ is from models testing the hypothesis that $\mathrm{OR}_{\mathrm{AG}}$ and $\mathrm{OR}_{\mathrm{AP}}$ are different. $\mathrm{AP}=\mathrm{activPAL} ; \mathrm{AG}=\mathrm{ActiGraph} ; \mathrm{BMI}=$ body mass index; $\mathrm{CI}=$ confidence interval; $\mathrm{OR}_{\mathrm{AP}}=$ odds ratio and $95 \% \mathrm{CI}$ for associations with $\mathrm{AP}$-measured variables; $\mathrm{OR}_{\mathrm{AG}}=\mathrm{odds}_{\text {ratio }}$ and $95 \% \mathrm{CI}$ for associations with AG-measured variables.

$\mathrm{AG}_{100 \mathrm{cpm}}$ detected significantly more breaks in sedentary time than did the AP, which resulted in shorter sedentary bouts, on average. Estimates of $A_{100 c p m}$ metrics thus reflected more frequently interrupted sedentary time accumulation than AP estimates, as shown by significantly shorter mean bout duration and UBD, and higher alpha. Studies that rely on $\mathrm{AG}_{100 \mathrm{cpm}}$ estimates of sedentary behavior patterns (e.g., descriptive epidemiology studies, dose-response analyses) should take these between-device differences into account, recognizing that $\mathrm{AG}_{100 \mathrm{cpm}}$-derived metrics systematically misestimate sedentary behavior patterns. This will be particularly important for researchers working to establish sedentary behavior guidelines as the values that might be considered "too sedentary" or "too prolonged of a sedentary behavior pattern" will differ depending on the device used. More studies are needed to quantify the between-device differences and their potential impact on future recommendations. Notably, the approximate $1 \mathrm{hr} /$ day difference in total sedentary time and time in $30+$ min bouts observed in this study highlight that the between-device differences are large enough that they could have important public health implications.

On the other hand, even with the between-device differences in estimates of sedentary behavior volume and patterns, we observed moderate to high linear relationships between sedentary behavior 
(a) Associations with self-rated health (good, poor, and very poor vs. very good and excellent)

\begin{tabular}{|c|c|c|c|c|c|}
\hline & $\mathrm{OR}_{\mathrm{AP}}$ & $\mathrm{OR}_{\mathrm{AG}}$ & \multicolumn{2}{|l|}{$p$ value $_{\text {Horton }}$} & ActivPaL $\square$ ActiGraph \\
\hline Total sedentary time & $1.30[1.13,1.50]$ & $1.27[1.06,1.51]$ & .15 & & $1 \longmapsto$ \\
\hline Time spent in bouts $\geq 30 \mathrm{~min}$ & $1.36[1.18,1.57]$ & $1.29[1.11,1.51]$ & .20 & & $1 \longmapsto$ \\
\hline Mean bout duration & $1.36[1.18,1.59]$ & $1.50[1.16,1.98]$ & $<.001$ & & $1 \longmapsto=1$ \\
\hline Usual bout duration & $1.33[1.15,1.56]$ & $1.38[1.12,1.72]$ & .01 & & $1 \longmapsto$ \\
\hline Average breaks in sed bouts & $0.87[0.75,1.00]$ & $0.88[0.79,0.97]$ & .05 & $r=$ & \\
\hline Alpha & $.85[.74, .98]$ & $.94[.90, .98]$ & $<.001$ & & \\
\hline & & & & 0.75 & $\begin{array}{lll}1.25 & 1.5 & 1.75\end{array}$ \\
\hline
\end{tabular}

(b) Associations with body mass index ( $\geq 30$ vs. $<30$ )

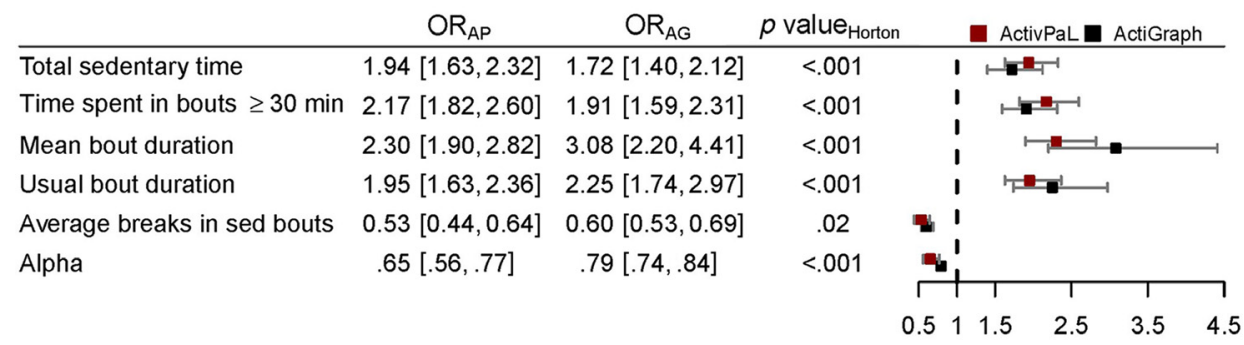

(c) Associations with difficulty walking half mile (any vs. none)

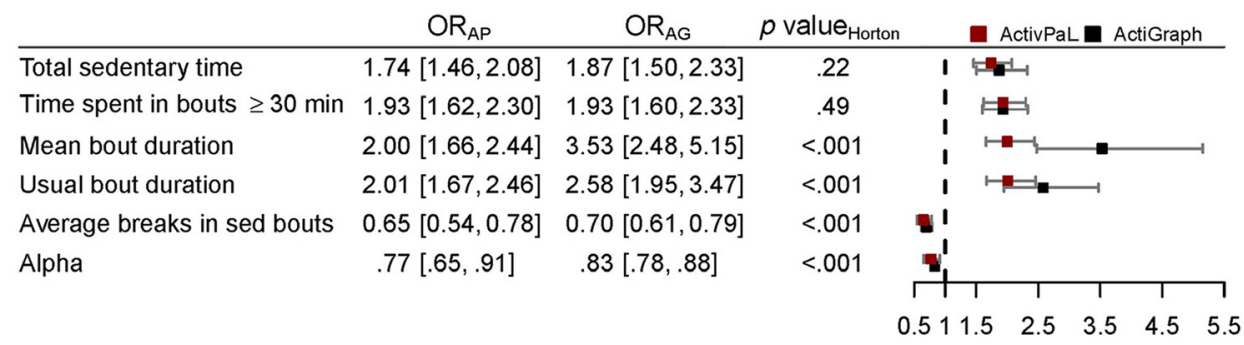

(d) Associations with cognitive impairment (impaired vs. not impaired)

\begin{tabular}{lcccc} 
& $\mathrm{OR}_{\mathrm{AP}}$ & $\mathrm{OR}_{\mathrm{AG}}$ & $p$ value \\
\hline Total sedentary time & $0.94[0.58,1.52]$ & $0.89[0.49,1.61]$ & .97 \\
Time spent in bouts $\geq 30 \mathrm{~min}$ & $1.13[0.71,1.76]$ & $1.13[0.70,1.78]$ & .83 \\
Mean bout duration & $1.26[0.90,1.66]$ & $1.56[0.96,2.32]$ & .53 \\
Usual bout duration & $1.17[0.84,1.51]$ & $1.45[0.92,2.08]$ & .66 \\
Average breaks in sed bouts & $0.86[0.55,1.33]$ & $0.91[0.68,1.24]$ & .76 \\
Alpha & $1.13[.70,1.88]$ & $.98[.84,1.13]$ & .11 & 0.5
\end{tabular}

Figure 2 - Forest plots for unstandardized associations between sedentary behavior measured using AG $100 \mathrm{cpm}$ and AP and self-rated health (a), BMI (b), difficulty walking half mile (c), and cognitive impairment (d). AP = activPAL; $\mathrm{AG}=\mathrm{ActiGraph}$; $\mathrm{BMI}=$ body mass index; $\mathrm{CI}=$ confidence interval; $\mathrm{CPM}=$ counts per minute; $\mathrm{OR}_{\mathrm{AP}}=$ odds ratio and $95 \% \mathrm{CI}$ for associations with $\mathrm{AP}$-measured variables; $\mathrm{OR}_{\mathrm{AG}}=\mathrm{odds}_{\mathrm{satio}}$ and $95 \% \mathrm{CI}$ for associations with AG-measured variables; USD $=$ usual bout duration. All models are adjusted for age, sex, race/ethnicity, and education. The $p$ value ${ }_{\text {Horton }}$ is from models testing the hypothesis that $\mathrm{OR}_{\mathrm{AG}}$ and $\mathrm{OR}_{\mathrm{AP}}$ are different. Odds ratios are unstandardized, meaning that for $\mathrm{AP}_{\text {and }} \mathrm{AG}_{100 \mathrm{cpm}}$, they are presented for the same increments for each sedentary behavior metric: $116 \mathrm{~min} /$ day for total sedentary time; $136 \mathrm{~min} /$ day for time in $30+$ min bouts; $6.6 \mathrm{~min}$ for mean bout duration; 17.8 for USD; 12.8 for breaks in sedentary behavior; and .04 for alpha.

metrics measured by $\mathrm{AG}_{100 \mathrm{cpm}}$ and $\mathrm{AP}$ (except for breaks in sedentary time and alpha), which suggest that metrics from either device similarly ranked participants according to high versus low sedentary time, and according to prolonged versus frequently interrupted sedentary behavior patterns. And when the metrics were first standardized, inferences of associations between sedentary behavior and health outcomes were not appreciably different for $\mathrm{AG}_{100 \mathrm{cpm}}$ versus $\mathrm{AP}$ measures. Associations have three main components: direction, magnitude, and statistical significance. $\mathrm{AG}_{100 \mathrm{cpm}}$ and $\mathrm{AP}$ similarities in the magnitude of associations were most apparent when sedentary behavior metrics were standardized (five associations out of 24 were significantly different) compared with when they were unstandardized (13 associations out of 24 were significantly different). Accuracy of the magnitude of associations are important because they enable more accurate computation of population attributable risk (or population attributable fraction), which are needed for efficiently allocating public health- and research-related resources. In addition and importantly, in all but one test, the statistical significance and the direction of associations were consistent between $\mathrm{AG}_{100 \mathrm{cpm}}$ and $\mathrm{AP}$ measures 
as illustrated by the $95 \%$ confidence intervals. Taken together, when interpreting associations using standardized (not unstandardized) metrics, the two devices resulted in generally similar inferences about the relationship of sedentary behavior with physical and cognitive health.

\section{Similar Findings From Other Studies}

As far as we know, the only other studies that evaluate agreement of $\mathrm{AG}_{100 \mathrm{cpm}}$ and AP measures of sedentary behavior patterns among adults focus on the metric "breaks in sedentary time." Breaks in sedentary time are the main component for measuring how sitting and sedentary time are accumulated because they define the beginning and end of each sedentary bout, and sedentary bouts are the underlying input for all sedentary behavior pattern metrics. In this study, we observed that significantly more breaks were counted by $\mathrm{AG}_{100 \mathrm{cpm}}$ compared with AP devices, leading to a high mean error and low concordance in the number of breaks measured per day, but a modest Pearson correlation coefficient of .51. These first findings among older adults, who accumulate sitting time differently than younger adults (Diaz et al., 2016), corroborate results from the previous studies discussed below that were conducted among adults (Barreira et al., 2015; Lyden et al., 2012) and children (Carlson et al., 2019).

Lyden et al. (2012) directly observed 13 participants between 20 and 60 years old (mean \pm SD age $25 \pm 5$ years) on two 10 -hr occasions, one during normal living, and another during a time when they were asked to reduce their sitting and to breakup sedentary time more frequently. The authors found that AP data provided accurate estimates of the number of breaks compared with direct observation. However, AG data, processed using the $100 \mathrm{cpm}$ cut point, overestimated breaks by more than twofold. Despite the differences in accuracy, the correlation between $A_{100 c p m}$ and direct observation was .86 during normal living and .64 during the treatment condition.

Barreira and colleagues compared breaks measured by AP to those measured by AG among 15 participants aged $28 \pm 3$ years. The number of $\mathrm{AG}_{100 \mathrm{~cm}}$ breaks were, on average, almost two times (90\%) higher than AP breaks (Barreira et al., 2015). Most of the additional $\mathrm{AG}_{100 \mathrm{cmm}}$ breaks $(52 \%)$ occurred when, according to the AP, participants were sitting. This demonstrates one source of error when measuring breaks in sedentary time using $A_{100 \mathrm{cpm}}$ : long sitting bouts are artificially broken into several shorter bouts when enough movement occurs (e.g., fidgeting or wiggling) that the $100 \mathrm{cpm}$ threshold is breached. Barreira et al. (2015) also reported that $40 \%$ of the additional $A_{100 \text { cpm }}$ breaks occurred while participants were standing. This demonstrates a second source of error when measuring breaks in sedentary time using $\mathrm{AG}_{100 \mathrm{cpm}} \cdot \mathrm{AG}_{100 \mathrm{cpm}}$ breaks reflect a transition from low levels of movement (i.e., movement below $100 \mathrm{cpm}$ ) to relatively more movement, and does not capture transitions in posture from sitting to standing. Therefore, $\mathrm{AG}_{100 \mathrm{cpm}}$ breaks can occur when a participant is standing with low level of movement then transitions to a higher level of movement (e.g., walking), or when they are engaging in a high level of movement and then stand sufficiently still before going back to a high level of movement. As this source of error will occur more often among people who stand more and standing has been associated with beneficial health outcomes (Katzmarzyk, 2014; Purva, 2021; Winkler et al., 2018), this might explain why, in this study, we observed marginally stronger associations with physical health outcomes for standardized $\mathrm{AG}_{100 \mathrm{cpm}}-$ measured breaks in sedentary time and alpha.
Among children, the most common accelerometer data processing protocol is to classify sedentary time using a 25 counts/15-s cut point. Using that protocol among 195 children aged 10.5 $(S D=0.7)$ years, Carlson et al. (2019) reported that $A_{25}$ counts detected a $307 \%$ mean absolute increase in the number of breaks compared with AP. While they reported that the respective mean absolute increase was just $25 \%$ when using $\mathrm{AG}_{100 \mathrm{cpm}}$, the interclass correlation between $\mathrm{AG}_{100 \mathrm{cpm}}$ and $\mathrm{AP}$ breaks was just .31. A deep dive into data revealed that despite similar averages $\left(\mathrm{AG}_{100 \mathrm{cpm}}=\right.$ $89.9 /$ day; $\mathrm{AP}=82.8 /$ day $)$, the timing of the breaks was very different between the devices, with the AP detecting breaks that went undetected by $\mathrm{AG}_{100 \mathrm{cpm}}$, and $\mathrm{AG}_{100 \mathrm{cpm}}$ detecting breaks that went undetected by the AP. Deviation in the timing of transitions resulted in a $57 \%$ and a $59 \%$ mean difference in UBD and alpha, respectively, and similar mean differences were observed in this study.

Similar to two smaller studies of agreement in measuring total sedentary time, $\mathrm{AG}_{100 \mathrm{cpm}}$ overestimated measures of daily sedentary time-by $55 \mathrm{~min} /$ day in this study compared with $107 \mathrm{~min} /$ day among 37 adults aged $74 \pm 7$ years (Aguilar-Farías et al., 2014) and to $114 \mathrm{~min} /$ day among 62 adults aged $78 \pm 6$ years (Koster et al., 2016). Like in the two previously mentioned studies, when alternate cut points were used, agreement with AP in total sedentary time improved and was underestimated by $45 \mathrm{~min} /$ day for $\mathrm{AG}_{200 \mathrm{cpm}}$ and $27 \mathrm{~min} /$ day $\mathrm{AG}_{25 \mathrm{cpm}}$.

\section{Strengths and Limitations}

This study has several strengths. Data were collected from a large community-based cohort study of older men and women. We included five different measures of sedentary behavior patterns as there remains a lack of consensus concerning which metric might be best. In addition, our criterion measure (AP) has been shown to be as good as direct observation for identifying breaks in sedentary behavior, which is requisite for measuring sedentary behavior patterns.

As in all studies, our results should be interpreted within the context of study limitations. Generalizability would be enhanced by introducing more racial/ethnic diversity and by including more geographic variability. Our AG sedentary behavior metrics were derived from data processed with the normal filter within ActiLife, as we do not yet have data processed using the low-frequency extension filter. However, at least one previous study among adults showed that agreement between sedentary breaks measured using $\mathrm{AG}_{100 \mathrm{cpm}}$ and direct observation is nearly the same when data were processed using the low-frequency extension filter or the normal frequency filter (Lyden et al., 2012). The Choi algorithm classifies periods of $90 \mathrm{~min}$ or more with zero movement (and requiring a 30-min streamframe and 2-min tolerance) as nonwear time. The 90-min duration and streamframe criteria were developed specifically for older adults and help prevent misclassifying sedentary time as nonwear time (Choi et al., 2012). However, it is possible that differences in sedentary behavior metrics between devices could have been impacted by using this data processing protocol, which is the most commonly used protocol among studies of older adults (Migueles et al., 2017). To test this, we repeated all analyses among the 596 adults who had four or more days without Choiidentified nonwear time, and the results were not appreciably changed (data not shown). While the AG GT3X+ is no longer sold by AG, the cross-generational accuracy with the currently available model (w-GT3X-BT) is exceptionally high (Miller, 2015). Finally, our study used data from the AP, which has very low error in comparison with gold standard direct observation (Lyden et al., 2012), but is not without error. 


\section{Conclusion}

This study showed that using $\mathrm{AG}_{100 \mathrm{cpm}}$ led to an overestimation of breaks in sedentary time and systematically more interrupted sedentary behavior patterns than were observed using AP measures. For example, mean bout duration as measured by AP was $66 \%$ longer, on average, than duration as measured by $A_{100 \mathrm{cpm}}$ and breaks in sedentary time measured by $A_{100 \mathrm{cpm}}$ was nearly twice as large. As a result, caution should be used when interpreting estimates of sedentary behavior patterns from $A_{100 \mathrm{cpm}}$ and any associations that rely on those pattern metric estimates. When our metrics were first standardized based on their underlying distribution-we used the $S D$, but an interquartile range or comparing quartiles of the exposure are also distribution-based methods that would work-associations with physical and cognitive health using $\mathrm{AG}_{100 \mathrm{cpm}}$ and $\mathrm{AP}$ were similar when the associations were interpreted using the distribution-based unit of analysis but not when the distribution-based units were converted back to the absolute unit of analysis. For example, we strongly recommend reporting results for a $1 S D$ increment in mean bout duration instead of an $x$-minute increment in mean bout duration, where $x$ is the $S D$ of the mean bout duration. Whenever estimates of associations are presented using absolute units of sedentary behavior pattern measures (e.g., minute, minute/day, n/day), the magnitude of the association will differ according to which device is used in determining those pattern measures. Consequently, if physical activity guidelines were developed using research conducted only by $\mathrm{AG}_{100 \mathrm{cpm}}$, the guidelines could be meaningfully different than if the same research were conducted using only AP. Future development of sedentary behavior guidelines will rely on accelerometer data from longitudinal studies. As many studies have already collected hip-worn AG data and many new studies will likely continue to employ the AG, research is needed to develop accelerometer data processing techniques (Kerr et al., 2018; Nakandala et al., 2021) or measurement error correction models (Sampson, Matthews, Freedman, Carroll, \& Kipnis, 2016) to increase the accuracy of sedentary behavior pattern metrics derived from hip-worn devices. Until then, for studies assessing associations of sedentary behavior volumes and patterns with health using $A_{100 \mathrm{cpm}}$, we recommend presenting results for standardized and unstandardized point estimates in $\mathrm{AG}_{100 \mathrm{cpm}^{-}}$ based studies and interpreting associations using units of the standardized metric rather than absolute units of sedentary behavior measures (e.g., minute, minute/day, n/day). This will increase accuracy of the relative magnitude of associations between sedentary behavior and health and might help harmonize results from prospective cohort studies that use different activity monitors. Ultimately, such an approach will help to answer the 2018 Physical Activity Guidelines Advisory Committee's call to provide more data about associations of sedentary behavior patterns, including bouts and breaks, with health outcomes (U.S. Department of Health and Human Services, 2018).

\section{Acknowledgments}

The authors have immense gratitude for the volunteers who took part in the Adult Changes in Thought (ACT) Study. This work was funded by the National Institute on Aging (U01 AG006781; DR and P01 AG052352; AZL) and the National Institute of Diabetes and Digestive and Kidney Diseases (R01 DK114945; LN). N.D. Ridgers is supported by a Future Leader Fellowship from the National Heart Foundation of Australia
(ID101895). The funders had no role in the design, conduct, analysis, and decision to publish results from this study.

\section{References}

Aguilar-Farías, N., Brown, W.J., \& Peeters, G.M.E.E.G. (2014). ActiGraph GT3X+ cut-points for identifying sedentary behaviour in older adults in free-living environments. Journal of Science and Medicine in Sport, 17(3), 293-299. PubMed ID: 23932934 doi:10.1016/j. jsams.2013.07.002

Barreira, T.V., Zderic, T.W., Schuna, J.M., Hamilton, M.T., \& TudorLocke, C. (2015). Free-Living activity counts-derived breaks in sedentary time: Are they real transitions from sitting to standing? Gait \& Posture, 42(1), 70-72. PubMed ID: 25953504 doi:10.1016/j. gaitpost.2015.04.008

Bellettiere, J., LaMonte, M.J., Evenson, K.R., Rillamas-Sun, E., Kerr, J., Lee, I.-M., ... LaCroix, A.Z. (2019). Sedentary behavior and cardiovascular disease in older women: The OPACH study. Circulation, 139(8), 1036-1046. PubMed ID: 31031411 doi:10.1161/ CIRCULATIONAHA.118.035312

Bellettiere, J., Winkler, E.A.H., Chastin, S.F.M., Kerr, J., Owen, N., Dunstan, D.W., \& Healy, G.N. (2017). Associations of sitting accumulation patterns with cardio-metabolic risk biomarkers in Australian adults. PLoS One, 12(6), e0180119. PubMed ID: 28662164 doi:10.1371/journal.pone.0180119

Bland, J.M., \& Altman, D.G. (2007). Agreement between methods of measurement with multiple observations per individual. Journal of Biopharmaceutical Statistics, 17(4), 571-582. PubMed ID: 17613642 doi:10.1080/10543400701329422

Brocklebank, L.A., Falconer, C.L., Page, A.S., Perry, R., \& Cooper, A.R. (2015). Accelerometer-Measured sedentary time and cardiometabolic biomarkers: A systematic review. Preventive Medicine, 76, 92-102. PubMed ID: 25913420 doi:10.1016/j.ypmed.2015.04.013

Carlson, J.A., Bellettiere, J., Kerr, J., Salmon, J., Timperio, A., Verswijveren, S.J.J.M., \& Ridgers, N.D. (2019). Day-level sedentary pattern estimates derived from hip-worn accelerometer cut-points in 8-12year-olds: Do they reflect postural transitions? Journal of Sports Sciences, 37(16), 1899-1909. PubMed ID: 31002287 doi:10.1080/ 02640414.2019 .1605646

Chastin, S.F.M., \& Granat, M.H. (2010). Methods for objective measure, quantification and analysis of sedentary behaviour and inactivity. Gait and Posture, 31(1), 82-86. PubMed ID: 19854651 doi:10.1016/ j.gaitpost.2009.09.002

Chastin, S.F.M., Winkler, E.A.H., Eakin, E.G., Gardiner, P.A., Dunstan, D.W., Owen, N., \& Healy, G.N. (2015). Sensitivity to change of objectively-derived measures of sedentary behavior. Measurement in Physical Education and Exercise Science, 19(3), 138-147. doi:10. 1080/1091367X.2015.1050592

Chiu, E.C., Yip, P.K., Woo, P., \& Lin, Y.Te. (2019). Test-retest reliability and minimal detectable change of the Cognitive Abilities Screening Instrument in patients with dementia. PLoS One, 14(5), e0216450. PubMed ID: 31063491 doi:10.1371/journal.pone.0216450

Choi, L., Liu, Z., Matthews, C.E., \& Buchowski, M.S. (2011). Validation of accelerometer wear and nonwear time classification algorithm. Medicine \& Science in Sports \& Exercise, 43(2), 357-364. PubMed ID: 20581716 doi:10.1249/MSS.0b013e3181ed61a3

Choi, L., Ward, S.C., Schnelle, J.F., \& Buchowski, M.S. (2012). Assessment of wear/nonwear time classification algorithms for triaxial accelerometer. Medicine \& Science in Sports \& Exercise, 44(10), 2009-2016. PubMed ID: 22525772 doi:10.1249/MSS.0b013e318258cb36 
De Oliveira, G.M., Yokomizo, J.E., E Silva, L.D.S.V., Saran, L.F., Bottino, C.M.C., \& Yassuda, M.S. (2016). The applicability of the cognitive abilities screening instrument-short (CASI-S) in primary care in Brazil. International Psychogeriatrics, 28(1), 93-99. PubMed ID: 25921381 doi:10.1017/S1041610215000642

Diaz, K.M., Goldsmith, J., Greenlee, H., Strizich, G., Qi, Q., MossavarRahmani, Y., ... Kaplan, R. (2017). Prolonged, uninterrupted sedentary behavior and glycemic biomarkers among US Hispanic/ Latino adults: The Hispanic Community Health Study/Study of Latinos (HCHS/SOL). Circulation, 136(15), 1362-1373. PubMed ID: 28835368 doi:10.1161/CIRCULATIONAHA.116.026858

Diaz, K.M., Howard, V.J., Hutto, B., Colabianchi, N., Vena, J.E., Blair, S.N., \& Hooker, S.P. (2016). Patterns of sedentary behavior in US middle-age and older adults: The REGARDS study. Medicine \& Science in Sports \& Exercise, 48(3), 430-438. PubMed ID: 26460633 doi:10.1249/MSS.0000000000000792

Diaz, K.M., Howard, V.J., Hutto, B., Colabianchi, N., Vena, J.E., Safford, M.M., ... Hooker, S.P. (2017). Patterns of sedentary behavior and mortality in U.S. middle-aged and older adults a national cohort study. Annals of Internal Medicine, 167(7), 465-475. PubMed ID: 28892811 doi:10.7326/M17-0212

Du, Y., Liu, B., Sun, Y., Snetselaar, L.G., Wallace, R.B., \& Bao, W. (2019). Trends in adherence to the physical activity guidelines for Americans for aerobic activity and time spent on sedentary behavior among US adults, 2007 to 2016. JAMA Network Open, 2(7), e197597. PubMed ID: 31348504 doi:10.1001/jamanetworkopen.2019.7597

Edwardson, C.L., Winkler, E.A.H., Bodicoat, D.H., Yates, T., Davies, M.J., Dunstan, D.W., \& Healy, G.N. (2016). Considerations when using the activPAL monitor in field based research with adult populations. Journal of Sport and Health Science, 6(2), 162-178. PubMed ID: 30356601 doi:10.1016/j.jshs.2016.02.002

Glazer, N.L., Lyass, A., Esliger, D.W., Blease, S.J., Freedson, P.S., Massaro, J.M., ... Vasan, R.S. (2013). Sustained and shorter bouts of physical activity are related to cardiovascular health. Medicine \& Science in Sports \& Exercise, 45(1), 109-115. PubMed ID: 22895372 doi:10.1249/MSS.0b013e31826beae5

Horton, N.J., \& Fitzmaurice, G.M. (2004). Regression analysis of multiple source and multiple informant data from complex survey samples. Statistics in Medicine, 23(18), 2911-2933. PubMed ID: 15344194 doi:10.1002/sim.1879

Jefferis, B.J., Parsons, T.J., Sartini, C., Ash, S., Lennon, L.T., Papacosta, O., ... Whincup, P.H. (2019). Objectively measured physical activity, sedentary behaviour and all-cause mortality in older men: Does volume of activity matter more than pattern of accumulation? British Journal of Sports Medicine, 53(16), 1013-1020. PubMed ID: 29440040 doi:10.1136/bjsports-2017-098733

Jefferis, B.J., Sartini, C., Shiroma, E., Whincup, P.H., Wannamethee, S.G., \& Lee, I.M. (2015). Duration and breaks in sedentary behaviour: Accelerometer data from 1566 community-dwelling older men (British Regional Heart Study). British Journal of Sports Medicine, 49(24), 1591-1594. PubMed ID: 25232029 doi:10.1136/bjsports2014-093514

Katzmarzyk, P.T. (2014). Standing and mortality in a prospective cohort of Canadian adults. Medicine \& Science in Sports \& Exercise, 46(5), 940946. PubMed ID: 24152707 doi:10.1249/MSS.0000000000000198

Katzmarzyk, P.T., Powell, K.E., Jakicic, J.M., Troiano, R.P., Piercy, K., \& Tennant, B. (2019). Sedentary behavior and health: Update from the 2018 physical activity guidelines advisory committee. Medicine \& Science in Sports \& Exercise, 51(6), 1227-1241. PubMed ID: 31095080 doi:10.1249/MSS.0000000000001935

Kerr, J., Carlson, J., Godbole, S., Cadmus-Bertram, L., Bellettiere, J., \& Hartman, S. (2018). Improving Hip-Worn Accelerometer Estimates of Sitting Using Machine Learning Methods Medicine \& Science in Sports \& Exercise, 50(7), 1518-1524. doi:10.1249/MSS.000000 0000001578

Koster, A., Shiroma, E., Caserotti, P., Mathews, C., Chen, K., Glynn, N., \& Harris, T. (2016). Comparison of sedentary estimates between activPAL and hip- and wrist-worn actiGraph. Medicine \& Science in Sports \& Exercise, 48(8), 1514-1522. PubMed ID: 27031744 doi:10. 1249/MSS.0000000000000924

Lee, I.-M., \& Shiroma, E.J. (2014). Using accelerometers to measure physical activity in large-scale epidemiological studies: Issues and challenges. British Journal of Sports Medicine, 48(3), 197-201. PubMed ID: 24297837 doi:10.1136/bjsports-2013-093154

Lord, S., Chastin, S.F.M., McInnes, L., Little, L., Briggs, P., \& Rochester, L. (2011). Exploring patterns of daily physical activity and sedentary behaviour in community-dwelling older adults. Age and Ageing, 40(2), 205-210. PubMed ID: 21239410 doi:10.1093/ageing/ afq166

Lyden, K., Kozey Keadle, S.L., Staudenmayer, J.W., \& Freedson, P.S. (2012). Validity of two wearable monitors to estimate breaks from sedentary time. Medicine \& Science in Sports \& Exercise, 44(11), 2243-2252. PubMed ID: 22648343 doi:10.1249/MSS.0b013e3 $18260 \mathrm{c} 477$

Matthews, C.E., George, S.M., Moore, S.C., Bowles, H.R., Blair, A., Park, Y., .. Schatzkin, A. (2012). Amount of time spent in sedentary behaviors and cause-specific mortality in US adults. American Journal of Clinical Nutrition, 95(2), 437-445. PubMed ID: 22218159 doi:10.3945/ajen.111.019620

Migueles, J.H., Cadenas-Sanchez, C., Ekelund, U., Nyström, C.D., MoraGonzalez, J., Löf, M., . . Ortega, F.B. (2017). Accelerometer data collection and processing criteria to assess physical activity and other outcomes: A systematic review and practical considerations. Sports Medicine, 47(9), 1821-1845. PubMed ID: 28303543 doi:10.1007/ s40279-017-0716-0

Miller, J. (2015). ActiGraph cross-generation accuracy confirmation. Retrieved from https://s3.amazonaws.com/actigraphcorp.com/wpcontent/uploads/2018/03/11163743/cross-generation-accuracy.pdf

Nakandala, S., Jankowska, M., Tuz-Zahra, F., Bellettiere, J., Carlson, J.A., .. Natarajan , L. (2021). Application of convolutional neural network algorithms for advancing sedentary and activity bout classification. Journal for the Measurement of Physical Behaviour. Advance online publication. doi:10.1123/JMPB.2020-0016

Purva, J., John, B., Nicole, G., Michael, J.L., Chongzhi, D., Robert, A.W., . . Andrea, Z.L. (2021). v. The Journals of Gerontology Series A, Biological Sciences and Medical Sciences, 76(1), 77-84. doi:10. 1093/gerona/glaa227

Rosenberg, D., Walker, R., Greenwood-Hickman, M.A., Bellettiere, J., Xiang, Y., Richmire, K., . . Lacroix, A.Z. (2020). Device-assessed physical activity and sedentary behavior in a community-based cohort of older adults. BMC Public Health, 20(1), 1256. PubMed ID: 32811454 doi:10.1186/s12889-020-09330-z

Sampson, J.N., Matthews, C.E., Freedman, L.S., Carroll, R.J., \& Kipnis, V. (2016). Methods to assess measurement error in questionnaires of sedentary behavior. Journal of Applied Statistics, 43(9), 1706-1721. doi:10.1080/02664763.2015.1117593

Teng, E.L., Hasegawa, K., Homma, A., Imai, Y., Larson, E., Graves, A., ... White, L.R. (1994). The Cognitive Abilities Screening Instrument (CASI): A practical test for cross-cultural epidemiological studies of dementia. International Psychogeriatrics, 6(1), 45-58. PubMed ID: 8054493 doi:10.1017/S1041610294001602

Tremblay, M.S., Aubert, S., Barnes, J.D., Saunders, T.J., Carson, V., Latimer-Cheung, A.E., ... Participants, on behalf of S. T. C. P. (2017). Sedentary Behavior Research Network (SBRN)- 
Terminology Consensus Project process and outcome. International Journal of Behavioral Nutrition and Physical Activity, 14(1), 75. PubMed ID: 28599680 doi:10.1186/s12966-017-0525-8

U.S. Department of Health and Human Services. (2018). Physical activity guidelines for Americans (2nd ed.). Retrieved from U.S. Department of Health and Human Services website: https://health.gov/paguide lines/second-edition/report/

Ware, C.J.E., \& Sherbourn, D. (1992). The MOS 36-item short-form health survey (SF-36): I. Conceptual framework and item selection. Medical Care, 30(6), 473-483. PubMed ID: 1593914 doi:10.1097/ 00005650-199206000-00002
Ware, J.E. (2000). SF-36 health survey update. Spine, 25(24), 3130-3139. PubMed ID: 11124729 doi:10.1097/00007632-200012150-00008

Winkler, E.A.H., Chastin, S., Eakin, E.G., Owen, N., Lamontagne, A.D., Moodie, M., ... Healy, G.N. (2018). Cardiometabolic impact of changing sitting, standing, and stepping in the workplace. Medicine \& Science in Sports \& Exercise, 50(3), 516-524. PubMed ID: 29166319 doi:10.1249/MSS.0000000000001453

Yang, L., Cao, C., Kantor, E.D., Nguyen, L.H., Zheng, X., Park, Y., ... Cao, Y. (2019). Trends in sedentary behavior among the US population, 2001-2016. JAMA, 321(16), 1587. PubMed ID: 31012934 doi:10.1001/jama.2019.3636 